Objectives: We undertook a pilot survey of regional GPs to understand their level of prescribing confidence with an aim to develop a targeted teaching program.

Methods: Based on BSR DMARD monitoring audit tool and regionally approved shared care guidelines, a focus group discussion with GP educators and CCG Medicine Optimisation Team was organised. Ten items were unanimously identified as core knowledge required for safe prescribing. A questionnaire was created based on these elements. GPs were surveyed at their educational meeting in our academic institution. Replies were compiled to ascertain their understanding of safe prescribing and troubleshooting DMARD related issues.

Results: There are 95 actively practicing GPs in the region. 41 (43\%) GPs contributed to the survey. 22 (56\%) were men. 27 (65\%) had been practicing for 5-15 years (range $<5$ to $>20$ years). 34 ( $83 \%$ ) regularly prescribed DMARDs. Only $15 /$ $41(36 \%)$ felt confident in prescribing whereas $7 / 41(17 \%)$ were neutral and remaining $46 \%$ did not feel confident. $49 \%$ participants were able to rightly answer questions pertaining to safe prescribing. Again most (70\%) felt unable to confidently manage blood-monitoring issues such as neutropenia or abnormal liver function tests. Only four ( $9 \%$ ) were able to correctly address the concerns related to administration of live vaccines whilst taking DMARDs. Nine GPs were unaware of avoiding trimethoprim co-prescription with methotrexate.

Conclusions: To our knowledge, this is the first survey to demonstrate that there are serious shortcomings in GPs' understanding of safe DMARD prescribing. Lack of confidence amongst front line medical staff remains the main cause of this issue. Despite frequent education bulletins and alerts from national bodies including NICE, BSR and NPSA and active measures taken in recent past to identify better ways to address the concerns, this study highlights major knowledge gaps among everyday prescribers in primary care. Dedicated strategy and better collaboration with GPs, with focus on drug monitoring education, are pivotal to providing better care for patients prescribed DMARDs in the community.

Disclosure of Interest: None declared

DOI: 10.1136/annrheumdis-2018-eular.1301

\section{FRI0696 PATIENT ACTIVATION AND ADHERENCE TO BIOLOGICAL THERAPY: PRELIMINARY RESULTS}

A. Sendra-García ${ }^{1,2,3}$, M. A. Portes-Peralta ${ }^{4}$, A. Moya-Gill ${ }^{1}$, J. P. Ordovás-Baines ${ }^{1}$, J. J. Alegre-Sancho ${ }^{2}$, M. Climente-Marti ${ }^{1}$. ${ }^{1}$ Pharmacy Department, ${ }^{2}$ Rheumatology Department, Dr. Peset University Hospital. Valencia, ${ }^{3}$ Foundation for the Promotion of Health and Biomedical Research of Valencia, FIsABIO, ${ }^{4}$ Pharmacology

Department, University of Valencia, Valencia, Spain

Background: Medication non-adherence is associated with treatment failure. Some authors show a positive relationship between patient activation and both, adherence to treatment for chronic conditions and improved outcomes.

Objectives: To present preliminary results of a study measuring adherence to biological therapy and its relationship with the Patient Activation Measure (PAM) in the outpatient setting.

Methods: Ambispective longitudinal observational descriptive study in a general tertiary university hospital.

Patients on treatment with the same biological drug for $\geq 6$ months were included in order of arrival. Patients with some degree of mental disability, which prevented understanding of the purpose and parameters of the study, were excluded.

Demographic variables (sex, age, environment, educational level), diagnosis and treatment were collected. To measure adherence, the Simplified Medication Adherence Questionnaire (SMAQ), validated in Spain, and the medication possession ratio (MPR) were used. Patients were considered as non-adherent if MPR $<80 \%$ and/ or non-adherent SMAQ. To measure patient's ability to play an active role in their health care, PAM questionnaire, consisting on 13 items and validated in Spain, was used. It sorts patients into 4 activation levels, which were grouped together into not activated (PAM 1-2) or activated (PAM 3-4).

Relationship between adherence to treatment, as a combined variable, and PAM was analyzed using chi-square, considering significance level $p<0,05$. Statistical analysis were performed with spss v17.0.

Results: Fifty patients (58\% women) were included. Mean age: 48 years (95\% Cl: 33 to 63 ); $92 \%$ lived in urban areas, $28 \%$ completed elementary education, $44 \%$ high-school and $28 \%$ university studies.

Diagnosis: rheumatoid arthritis $(38 \%)$, Crohn's disease $(20 \%)$, psoriasis $(20 \%)$, ankylosing spondylitis (16\%) and psoriatic arthritis (6\%). Treatment: adalimumab $(44 \%)$, etanercept $(16 \%)$, tocilizumab $(16 \%)$, secukinumab $(12 \%)$, ustekinumab $(6 \%)$, golimumab (4\%) and ixekizumab (2\%). Median time on the biological drug treatment: 26 months (IQR 53).

PAM level: 2, 16, 54 and $28 \%$ for levels $1,2,3$ and 4, respectively.
Table 1 Global distribution and relationship between adherence and PAM

\begin{tabular}{|c|c|c|c|c|}
\hline \multirow[t]{3}{*}{$\mathrm{n}(\%)$} & \multicolumn{3}{|c|}{ Adherent patients } & \multirow[t]{3}{*}{ Total } \\
\hline & SMAQ & MPR & Combined & \\
\hline & $38(76 \%)$ & $29(59 \%)^{*}$ & $23(47 \%)^{\star}$ & \\
\hline \multicolumn{5}{|c|}{ Activated patients } \\
\hline Yes & 32 (78\%) & $25(63 \%)^{*}$ & $20(50 \%)^{*}$ & $41(82 \%$ \\
\hline No & $6(67 \%)$ & $4(44 \%)^{*}$ & $3(33 \%)^{*}$ & $9(18 \%)$ \\
\hline$\chi^{2} ; p$ & 0,$524 ; 0,668$ & 0,$991 ; 0,456$ & 0,$819 ; 0,472$ & \\
\hline
\end{tabular}

*1 lost patient.

The proportion of adherent patients was $47 \%$ (23/49), being higher (50\%) among the activated patients compared to the non-activated patients (33\%), even though the differences were not statistically significant

Conclusions: Among biologic treated patients, $82 \%$ show a high degree of activation on their disease and treatment self-management. However, only $47 \%$ were adherent to treatment, when combining the SMAQ questionnaire and the medication possession ratio quantification.

The greater proportion of adherence found among patients with a higher degree of activation could indicate a positive relationship between activation and adherence, so analyzing and promoting patient activation seems important in order to improve adherence to biologic drugs.

Disclosure of Interest: None declared

DOI: 10.1136/annrheumdis-2018-eular.6508

\section{FRI0697 EXPECTATIONS, MOTIVATING FACTORS AND BARRIERS TO BASIC RESEARCH AMONG YOUNG RHEUMATOLOGISTS IN GERMANY- RESULTS OF A NATIONAL ONLINE SURVEY}

J. Mucke ${ }^{1}$, M. Schneider ${ }^{1}$, U. Wagner ${ }^{2}$, H. Schulze-Koops ${ }^{3}$, A. Skapenko ${ }^{3}$ J. Leipe ${ }^{3}{ }^{1}$ Policlinic for Rheumatology, Heinrich-Heine-University, Duesseldorf, ${ }^{2}$ Department of Rheumatology, University Hospital, Leipzig, ${ }^{3}$ Division of Rheumatology and Clinical Immunology, Department of Internal Medicine IV, University of Munich, Germany

Background: In Germany, the number of clinical fellows, who decide for a scientific career in basic research, is decreasing, leading to a growing lack of junio physician scientists (PS) highly needed to advance basic research. ${ }^{1}$ Structured mentoring and funding programs as well as other incentives might be necessary to attract young physicians and support starting a scientific career in basic science.

Objectives: To identify expectations, motivating factors and barriers of final year medical students interested in rheumatology, of fellows and of physicians in rheumatology to start a scientific career in basic research.

Methods: An online survey was distributed by email and via facebook and twitte to members of the German Network of Young Rheumatologists (rheumadocs) medical students in their final year participating in a student program of the German Society for Rheumatology (DGRh), and by direct contact to PS. The questions focused on demographics, scientific background (including experience in basic research), expectations such as scientific self-realization, acquirement of a postdoctoral degree, enhanced career options, as well as motivating factors (e.g "fun", financial benefits, travel bursaries, mentoring programs and others) and barriers (e.g. positions in basic science, exemption from clinical duties, lack of experience and/or assistance in fund-raising and scientific methods) with regard to a career in basic research.

Results: Of the 91 responders, $75 \%$ was aged below 35 years $(11 \%<25$ yrs $32 \% 25-29$ yrs, $57 \%>29$ yrs) and 58\% were female. $19 \%$ were currently working in basic research. $23 \%$ were students. $35 \%$ were fellows currently in the clinic. $23 \%$ were primary attendants. Basic research experience was reported by $49 \%$ of all clinicians. Only $10 \%$ indicated not to be interested in performing basic research. Among the students, $48 \%$ were currently performing a laboratory-based medical thesis. Of interest, no substantial differences in expectations or barriers were found between students, PS and clinicians. As to motivational factors, PS named time spent in research accounted for rheumatology training $(74 \%)$, support by excellence programs $(71 \%)$ and enhanced career options with a postdoctora degree $(65 \%)$ more often than clinicians and students $(57 \%, 50 \%$ and $42 \%$ respectively). Other common motivational factors were "fun" (76\%) and travel bursaries for international meetings (62\%). Financial benefits in terms of standard or even senior physician salaries were no major motivators $(41 \%$ and $24 \%$, respectively). Regarding expectations, $71 \%$ indicated scientific self-realization, $58 \%$ named enhanced career options. $78 \%$ indicated lacking exemption from clinical 